\title{
A Comparison of Haar Wavelets and Kekre's Wavelets for Storing Colour Information in a Greyscale Image
}

\author{
Dr. H. B. Kekre \\ Senior Professor \\ Computer Engg. Department, \\ Mukesh Patel School of \\ Technology Management and \\ Engineering, \\ SVKM's NMIMS University \\ Mumbai, India
}

\author{
Sudeep D. Thepade \\ Associate Professor, \\ Computer Engg. Department, \\ Mukesh Patel School of \\ Technology Management and \\ Engineering, \\ SVKM's NMIMS University \\ Mumbai, India
}

\author{
Adib Parkar \\ Thadomal Shahani Engineering \\ College \\ Mumbai University \\ Mumbai, India
}

\begin{abstract}
The technique to store colour information within a greyscale image has been proposed previously using various colour spaces, such as the YCbCr, LUV, YCgCb, YUV, and YIQ colour spaces, and using the Haar wavelet transform. One such study is described in [1]. This research paper extends the study to another wavelet transform - Kekre's wavelet transform (abbreviated as KWT). The embedding of the colour information into the greyscale image leads to the formation of a slightly distorted greyscale image known as a "matted greyscale" image. This matted greyscale image is used to reconstruct the colour image.
\end{abstract}

Keywords - Colouring, Colour to Grey, Matted Greyscale, Grey to Colour, Colour Space, YCbCr, LUV, YCgCb, YIQ, YUV, Haar Wavelets, Kekre's Wavelet Transform.

\section{INTRODUCTION}

Embedding colour information in a greyscale image has many advantages, not least of which is the fact that the colour image can be generated from just the greyscale image. This would simplify transmission of images as only one plane needs to be sent (i.e. the greyscale or luminance plane) instead of three planes, one each for red $(R)$, green $(G)$, and blue $(B)$.

One technique for achieving this is by transforming the colour image from the RGB colour space to a colour space that is based on luminance and chrominance components such as the $\mathrm{YCbCr}$ or LUV colour spaces. The luminance component is then taken to create the greyscale image which is transformed using some appropriate image transform such as the Discrete Wavelet Transform (DWT) or the Haar Transform. The chrominance components are then scaled down and are used to replace the lesser significant portions of the transformed matrix. This matrix is then inverse transformed back into a greyscale image, thus embedding colour information in the greyscale image.

This technique has been described in [1] using the $\mathrm{YCbCr}$, LUV, YCgCb, YUV, and YIQ colour spaces and the Haar transform. The technique itself is based on a technique proposed in [2] which used just the $\mathrm{YCbCr}$ colour space and the Discrete Wavelet Transform (abbreviated as DWT). This paper extends the study of the same technique by using a new transform - Kekre's Wavelet Transform (abbreviated as KWT) - and compares the results obtained using the Haar Transform and KWT across the same colour spaces.

The following sections describe the various colour spaces used, followed by details on the two transformations used (the Haar Transform and Kekre's Wavelet Transform). Subsequent sections provide a detailed description of the technique used to embed the colour information in the greyscale image, and finally the results of applying the technique using the different transforms and the different colour spaces. Hence a thorough comparison of the performance of this technique using the Haar Transform and
Kekre's Wavelet Transform is obtained across various colour spaces.

\section{YCbCr COLOUR SPACE}

The YCbCr model defines a colour space in terms of one luminance (brightness) and two chrominance (colour) components. It is one of the most extensively used colour spaces and has been considered for many applications such as those described in [2], [3], and [4]. In the $\mathrm{YCbCr}$ colour space, the Y component gives luminance and the $\mathrm{Cb}$ and $\mathrm{Cr}$ components give the chromaticity values of the colour image. To get the $\mathrm{YCbCr}$ components, the conversion of the RGB components to $\mathrm{YCbCr}$ components must be known. The RGB to $\mathrm{YCbCr}$ conversion matrix is given below.

$$
\left[\begin{array}{c}
Y \\
C b \\
C r
\end{array}\right]=\left[\begin{array}{ccc}
0.2989 & 0.5866 & 0.1145 \\
-0.1688 & -0.3312 & 0.5 \\
0.5 & -0.4184 & -0.0816
\end{array}\right] \cdot\left[\begin{array}{l}
R \\
G \\
B
\end{array}\right]
$$

To get the RGB values from the YCbCr components, the following conversion matrix can be used.

$$
\left[\begin{array}{l}
R \\
G \\
B
\end{array}\right]=\left[\begin{array}{ccc}
1 & -0.001 & 1.402 \\
1 & -0.3441 & -0.714 \\
1 & 1.7718 & 0.001
\end{array}\right] \cdot\left[\begin{array}{c}
Y \\
C b \\
C r
\end{array}\right]
$$

\section{LUV COLOUR SPACE}

The LUV colour space is a colour space generally used in techniques involving the colourization of images such as those described in [5], [6], [7], and [8]. In the LUV colour space, the L component provides the luminance, while the $\mathrm{U}$ and $\mathrm{V}$ components contain the colour information. The RGB to LUV conversion matrix is given below.

$$
\left[\begin{array}{l}
L \\
U \\
V
\end{array}\right]=\left[\begin{array}{ccc}
0.33333 & 0.33333 & 0.33333 \\
-0.3333 & 0.16667 & 0.16667 \\
0 & -0.5 & 0.5
\end{array}\right] \cdot\left[\begin{array}{l}
R \\
G \\
B
\end{array}\right]
$$

To get the RGB values from the LUV components, the following conversion matrix can be used.

$$
\left[\begin{array}{l}
R \\
G \\
B
\end{array}\right]=\left[\begin{array}{ccc}
1 & -2 & 0 \\
1 & 1 & -1 \\
1 & 1 & 1
\end{array}\right] \cdot\left[\begin{array}{l}
L \\
U \\
V
\end{array}\right]
$$

A negative value for the $\mathrm{U}$ component in the LUV colour space indicates prominence of the red component in the colour image. Similarly, a negative value for the $\mathrm{V}$ component indicates prominence of the green component over the blue component in the colour image. 


\section{YCgCb COLOUR SPACE}

The $\mathrm{YCgCb}$ colour model is a newly proposed colour space similar to the LUV colour space described in the previous section. Since it is newer than the LUV colour space, it has not yet been used extensively. One application not directly related to the technique under discussion is described in [9].

In the $\mathrm{YCgCb}$ colour space, the $\mathrm{Y}$ component provides the luminance, while the $\mathrm{Cg}$ and $\mathrm{Cb}$ components contain the chromaticity values. The $\mathrm{RGB}$ to $\mathrm{YCgCb}$ conversion matrix is given below.

$$
\left[\begin{array}{c}
Y \\
C g \\
C b
\end{array}\right]=\left[\begin{array}{ccc}
0.33333 & 0.33333 & 0.33333 \\
0.33333 & -0.3333 & 0 \\
0.33333 & 0 & -0.3333
\end{array}\right] \cdot\left[\begin{array}{l}
R \\
G \\
B
\end{array}\right]
$$

To get the RGB values from the $\mathrm{YCgCb}$ components, the following conversion matrix can be used.

$$
\left[\begin{array}{l}
R \\
G \\
B
\end{array}\right]=\left[\begin{array}{ccc}
1 & 1 & 1 \\
1 & -2 & 1 \\
1 & 1 & -2
\end{array}\right] \cdot\left[\begin{array}{c}
Y \\
C g \\
C b
\end{array}\right]
$$

A negative value for the $\mathrm{Cg}$ component in the $\mathrm{YCgCb}$ colour space indicates prominence of the green component over the red component in the colour image. Similarly, a negative value for the $\mathrm{Cb}$ component indicates prominence of the blue component over the red component.

\section{YIQ COLOUR SPACE}

YIQ is the colour space used by the NTSC colour TV system, employed mainly in North and Central America. 'I' stands for "in phase" and 'Q' stands for "quadrature," referring to the components used in quadrature amplitude modulation.

As in the $\mathrm{YCbCr}$ colour space, the $\mathrm{Y}$ component gives luminance and the $\mathrm{I}$ and $\mathrm{Q}$ components give the chromaticity values of the colour image. To get the YIQ components, the conversion of the RGB components to the YIQ components is defined by the following conversion matrix.

$$
\left[\begin{array}{l}
Y \\
I \\
Q
\end{array}\right]=\left[\begin{array}{ccc}
0.299 & 0.587 & 0.114 \\
0.596 & -0.275 & -0.321 \\
0.212 & -0.523 & 0.311
\end{array}\right] \cdot\left[\begin{array}{l}
R \\
G \\
B
\end{array}\right]
$$

To get the RGB values from the YIQ components, the following conversion matrix can be used.

$$
\left[\begin{array}{l}
R \\
G \\
B
\end{array}\right]=\left[\begin{array}{ccc}
1 & 0.956 & 0.621 \\
1 & -0.272 & -0.647 \\
1 & -1.107 & 1.704
\end{array}\right] \cdot\left[\begin{array}{l}
Y \\
I \\
Q
\end{array}\right]
$$

\section{YUV COLOUR SPACE}

YUV is a colour space that encodes a colour image or video taking human perception into account, allowing reduced bandwidth for chrominance, thereby typically enabling transmission errors or compression artefacts to be more efficiently masked by human perception than using a direct RGB representation.

Similar to the other colour spaces discussed previously, the $\mathrm{Y}$ component gives luminance, and the $\mathrm{U}$ and $\mathrm{V}$ components provide the chrominance values. The RGB to YUV conversion matrix is shown below.

$$
\left[\begin{array}{l}
Y \\
U \\
V
\end{array}\right]=\left[\begin{array}{ccc}
0.299 & 0.587 & 0.114 \\
-0.1471 & -0.2889 & 0.436 \\
0.615 & -0.5149 & 0.10001
\end{array}\right] \cdot\left[\begin{array}{l}
R \\
G \\
B
\end{array}\right]
$$

To get the RGB values from the YUV components, the following conversion matrix can be used.

$$
\left[\begin{array}{l}
R \\
G \\
B
\end{array}\right]=\left[\begin{array}{ccc}
0.74952 & -0.509 & 1.1398 \\
1.0836 & -0.2247 & -0.5806 \\
0.97086 & 1.9729 & 0.00001467
\end{array}\right] \cdot\left[\begin{array}{c}
Y \\
U \\
V
\end{array}\right]
$$

\section{HAAR TRANSFORM}

The Haar functions were proposed as a sequence in 1909 by Alfréd Haar [10]. Haar used these functions to give an example of a countable orthonormal system for the space of square-integrable functions on the real line. The study of wavelets, and even the term "wavelet", did not come until much later [11]. The Haar wavelet is also the simplest possible wavelet. As the Haar wavelet is not continuous, it is also not differentiable. This is a technical disadvantage of Haar wavelets.

The Haar wavelet's mother wavelet function $\psi(\mathrm{t})$ can be described as follows.

$$
\psi(t)=\left\{\begin{array}{cc}
1, & 0<t \leq \frac{1}{2} \\
-1, & \frac{1}{2}<t \leq 1 \\
0, & \text { otherwise }
\end{array}\right.
$$

\section{KEKRE'S WAVELET TRANSFORM}

The KWT matrix is a generic version of the LUV colour space matrix. Unlike most other transforms (wavelet or otherwise), the size of the KWT matrix need not be a power of two which is definitely an advantage of this transform.

The general form of an N x N KWT matrix is as follows.

$$
\left[\begin{array}{cccccc}
1 & 1 & 1 & \ldots & 1 & 1 \\
-N+1 & 1 & 1 & \ldots & 1 & 1 \\
0 & -N+2 & 1 & \ldots & 1 & 1 \\
\vdots & \vdots & \vdots & \ddots & \vdots & \vdots \\
0 & 0 & 0 & \ldots & 1 & 1 \\
0 & 0 & 0 & \ldots & -N+(N-1) & 1
\end{array}\right]
$$

Figure 1 - General N x N KWT matrix

As can be seen in figure 1 above, in the KWT matrix, all values above the diagonal are one (including the diagonal itself). The diagonal just below the primary diagonal has specific values, and all remaining values in the matrix are zeroes. In general, the value of $\mathrm{K}_{\mathrm{xy}}$ in the KWT matrix, where ' $\mathrm{x}$ ' is the row number starting from 1 and ' $y$ ' is the column number also starting from one, is given by the following equation.

$$
K_{x y}=\left\{\begin{array}{c}
1, \quad x \leq y \\
-N+(x-1), \quad x=y+1 \\
0, \quad \text { otherwise }
\end{array}\right.
$$

The inverse KWT matrix is nothing but the transpose of the above general matrix as the KWT is orthogonal. Normalization is required to get back the identity matrix.

\section{COLOUR TO MATTED GREY CONVERSION}

The most trivial way to convert a colour image to greyscale for printing is to retain and use the luminance component of the colour image. The problem with this approach is that regions that have contrasting colours with similar luminance components would be assigned the same output luminance level and would, therefore, look the same.

The other option is to map colours to textures [2]. One can control halftone dots or patterns as a function of the colours, for example, as a function of hue and saturation. Hence, regions of different colours with similar luminance will look different after mapping because they would have different textures [12]. The 
procedure proposed in [2] produces a continuum of textures using the DWT that naturally switch between patterns without causing visual artefacts. The DWT decomposes an image into several subbands [13] each representing different spatial frequency contents. In this paper, as explained previously, is an extension of [1] and is a more detailed study of a technique based on the one described in [2]. Here, the wavelet transforms used are the basic Haar transform and the KWT. Five different colour spaces are considered - LUV, $\mathrm{YCbCr}, \mathrm{YCgCb}, \mathrm{YIQ}$, and YUV. The greyscale image obtained by following the procedure outlined below is known as the matted greyscale image and it contains the colour information about the image embedded within its transform.

As various colour spaces are used, the procedure is outlined for a general colour space ' $A B C$ ' where ' $A$ ' is the luminance component, and ' $\mathrm{B}$ ' and ' $\mathrm{C}$ ' are the chromaticity components. As two different transforms are used, even the transforms are generalized as just 'the transform.' The following procedure has been taken from [1]. follows.

The steps involved to create the matted greyscale image are as

1. The original colour image is converted from the RGB colour space into the $\mathrm{ABC}$ colour space using the appropriate conversion matrix, that is, using equations (1), (3), (5), (7), or (9) for the YCbCr, LUV, YCgCb, YIQ, and YUV colour spaces respectively. The 'A' component is the luminance and is considered as the "original greyscale" image.

2. The ' $A$ ' component is transformed using the transform into the transform domain. Let this transformed image be known as $\mathrm{T}_{\mathrm{o}}$.

3. $\mathrm{T}_{\mathrm{o}}$ can be divided into 4 regions as shown in figure 1 below.

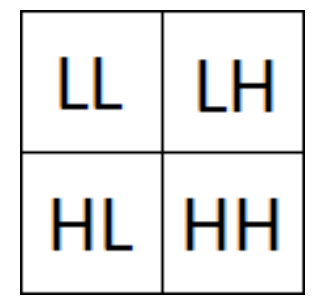

Figure 2 - Structure of $\mathrm{T}_{\mathrm{o}}$

4. Most of the information in the image is found in the LL region of $\mathrm{T}_{\mathrm{o}}$ which corresponds to the low frequency components of the image. This region is left untouched. The LH and HL regions of $T_{0}$ are replaced by scaled down versions of the ' $\mathrm{B}$ ' and ' $C$ ' components respectively. Thus we now have a modified transformed image that contains scaled down versions of the chromaticity components of the colour space being used. Let this be known as $\mathrm{T}_{\mathrm{m}}$.

5. The inverse transform is now applied to $T_{m}$ to get a new greyscale image in the spatial domain. This greyscale image now contains colour information hidden within its transform and is known as the matted greyscale image.

\section{COLOUR EXTRACTION FROM MATTED GREY}

Since the matted greyscale image already contains the colour information hidden within its transform, extracting the colour image from the matted greyscale image is a straightforward procedure. It consists of the following steps.

1. The matted greyscale image is read or scanned

2. Once available in digital form, the matted greyscale image is transformed using the transform into the transform domain. The transformed image obtained will be $\mathrm{T}_{\mathrm{m}}$.

3. $\mathrm{T}_{\mathrm{m}}$ can be represented as shown in figure 2 below.

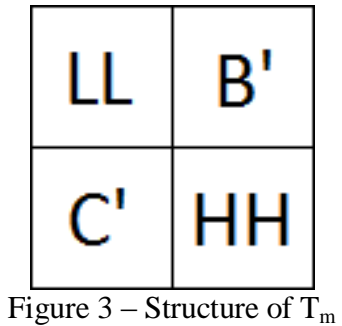

4. The $\mathrm{B}^{\text {' and }} \mathrm{C}^{\prime}$ regions of $\mathrm{T}_{\mathrm{m}}$ are the scaled down versions of the ' $\mathrm{B}$ ' and ' $\mathrm{C}$ ' components of the original colour image. Thus by extracting these two regions from $\mathrm{T}_{\mathrm{m}}$ and scaling them up back to their original size we get $B_{\text {approx }}$ and $C_{\text {approx }}$ which are approximations of the original ' $\mathrm{B}$ ' and ' $\mathrm{C}$ ' components of the original colour image.

5. To retrieve an approximation for the ' $A$ ' component, we replace regions $\mathrm{B}^{\prime}$ and $\mathrm{C}^{\prime}$ in $\mathrm{T}_{\mathrm{m}}$ by zeroes and perform an inverse transformation. The image obtained in the spatial domain is an approximation of the original ' $A$ ' component of the image, $\mathrm{A}_{\text {approx }}$.

Now the approximations for the $\mathrm{ABC}$ components are used to convert the image back to the RGB colour space using the appropriate conversion matrices, that is, equations (2), (4), (6), (8), and (10) for the YCbCr, LUV, YCgCb, YIQ, and YUV colour spaces respectively.

\section{IMPLEMENTATION AND RESULTS}

The implementation of the technique described in the previous section was an extension of the implementation described in [1]. The technique was broadened to include the KWT and the results obtained using the KWT were also added. The technique was applied to a large number of images. All the images were of the size $256 \times 256$ pixels, and belonged to various categories such as people, objects, vehicles, animals, cartoons, and nature.

On applying the technique proposed, the following set of images was derived from the each original image - original colour, original greyscale, matted greyscale, and reconstructed colour.

Performance was measured by calculating the mean square error (MSE) between the original greyscale image and the matted greyscale image as well as between the original colour image and the reconstructed colour image. The MSE is the mean of the square of the Euclidean distances between each pixel value. The Euclidean distance ' $d$ ' between two images $I_{m}$ and $I_{o}$ is defined as follows.

$$
d=\sqrt{\sum\left(z_{m}-z_{o}\right)^{2}}
$$

Where, $z_{m}$ is the value of the pixel in $I_{m}, z_{o}$ is the value of the corresponding pixel in $\mathrm{I}_{\mathrm{o}}$ and the summation is over all pixels in the images.

It must be noted that for a colour image, each pixel will have 3 values, one for the red $(R)$ plane, one for the green $(G)$ plane, and one for the blue (B) plane. The MSE thus provides an objective criterion that can be used as a measure of the degree of similarity between two given images. The greater the similarity between the two images at the pixel level, the lower the MSE.

The table 1 given below shows the MSE values between the matted greyscale images and the original greyscale images averaged across all images for each of the five colour spaces and both transforms. 


\begin{tabular}{|c|c|c|}
\hline & MSE (Haar) & MSE (KWT) \\
\hline YCbCr & 411.221335 & 401.618715 \\
\hline LUV & 341.32406 & 335.38614 \\
\hline YCgCb & 317.00611 & 309.915005 \\
\hline YIQ & 432.70043 & 423.13666 \\
\hline YUV & 643.38025 & 603.0701 \\
\hline
\end{tabular}

Table 1 - Greyscale MSE across all images

Figure 4 illustrates the results graphically.

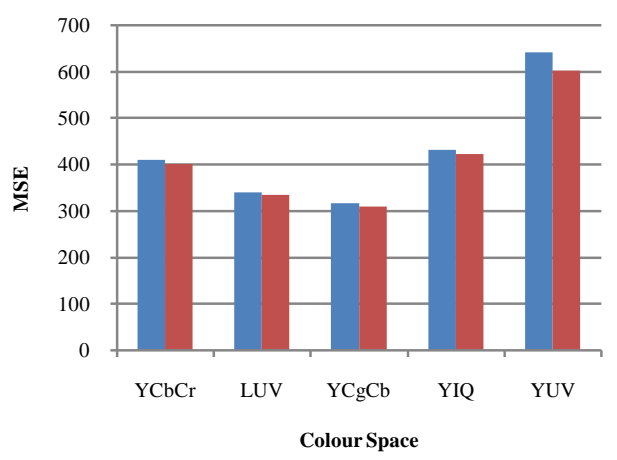

- MSE (Haar)

MSE (KWT)

Figure 4 - Greyscale MSE across all images

As can be clearly seen from the figure above, the $\mathrm{YCgCb}$ colour space provided the best results in terms of the MSE across all images for the matted greyscale image. Also, the KWT consistently outperformed the Haar transform across all colour spaces, but not by an extremely significant amount.

The results for the reconstructed colour images, however, were slightly different. The table below shows the MSE values between the reconstructed colour images and the original colour images.

\begin{tabular}{|c|c|c|}
\hline & MSE (Haar) & MSE (KWT) \\
\hline YCbCr & 245.36608 & 268.69903 \\
\hline LUV & 233.00122 & 249.32088 \\
\hline YCgCb & 245.31157 & 263.90563 \\
\hline YIQ & 249.90365 & 265.723 \\
\hline YUV & 417.922255 & 498.013915 \\
\hline
\end{tabular}

Table 2 - Colour MSE across all images

Figure 5 illustrates the results graphically.

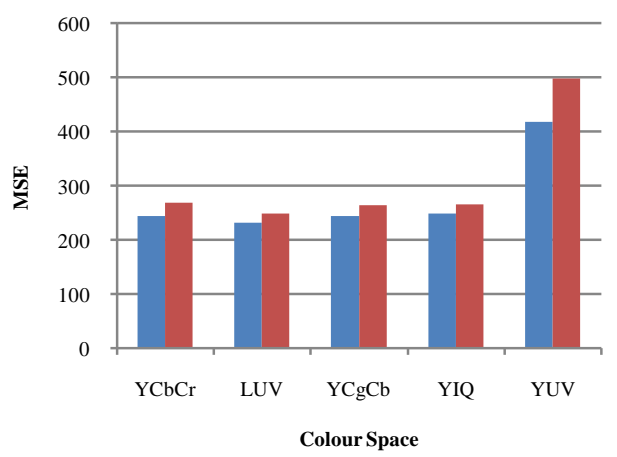

- MSE (Haar)

MSE (KWT)

Figure 5 - Colour MSE across all images
It can be noticed that in the case of the reconstructed colour images, there is no clear winner across four colour spaces when the MSE is averaged across all images: $\mathrm{YCbCr}, \mathrm{LUV}, \mathrm{YCgCb}$, and YIQ. The YUV colour space clearly underperforms using this technique for both greyscale and colour images and hence is not recommended. The colour space that performs the best is the LUV colour space, but not by a large amount. The $\mathrm{YCgCb}$ colour space, the YIQ colour space, and the $\mathrm{YCbCr}$ colour space give almost identical performance results based on the MSE.

When considering the transforms, on the other hand, here the roles are reversed. The Haar transform now appears to perform consistently better than the KWT. Once again, as the case was with the greyscale MSE, the difference in performance between the two transforms is now extremely significant.

Some of the images used when implementing this procedure are shown below.
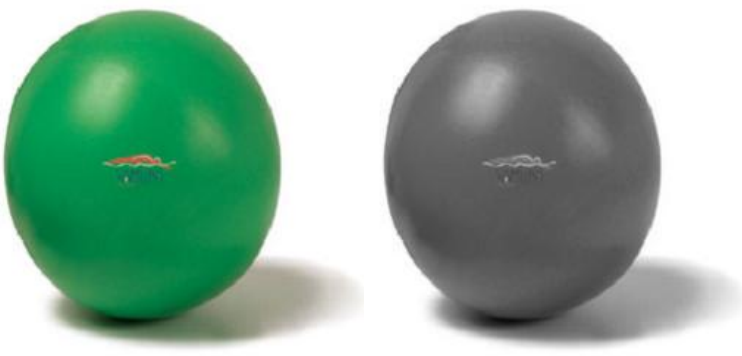

Figure 6a, b - Original Colour, Original Greyscale
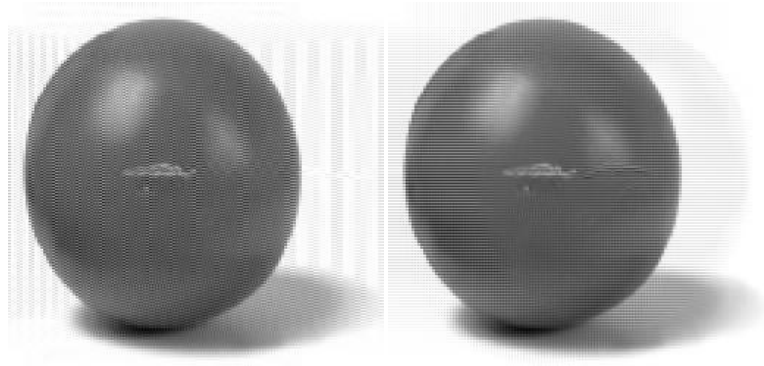

Figure 6c, d-Matted Greyscales (Haar, KWT)
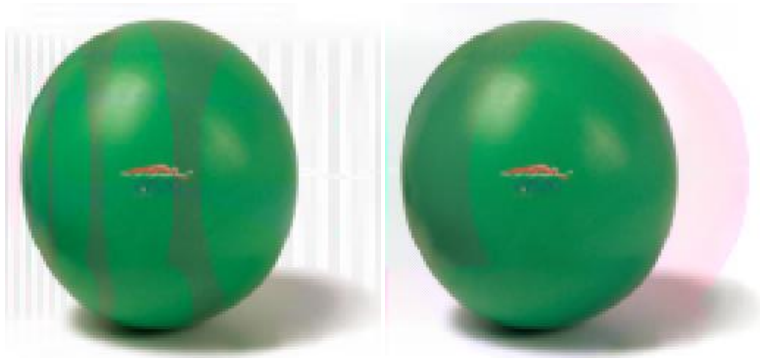

Figure 6e, f - Reconstructed Colour (Haar, KWT)

Figure 6 shows an image of a green ball. This is a simple image with a plain white background. The only object in the image is the ball itself. It can be seen that in the case of the matted greyscale image generated using the KWT, there appears to be an extra shadow behind the ball itself. While this shadow effect does not seem to unduly mar the quality of the matted greyscale image, the reconstructed colour image gets adversely affected.

Considering the Haar transform, the reconstructed colour image does not have the shadow effect behind the ball, however the ball itself is more discoloured than in the case of the KWT. There are at least three prominent discolourations on the green surface. 

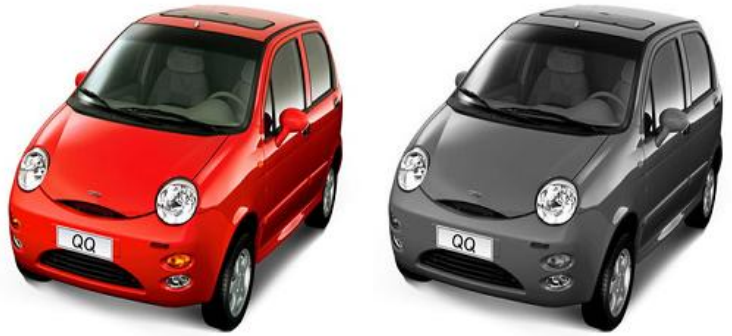

Figure 7a, b - Original Colour, Original Greyscale
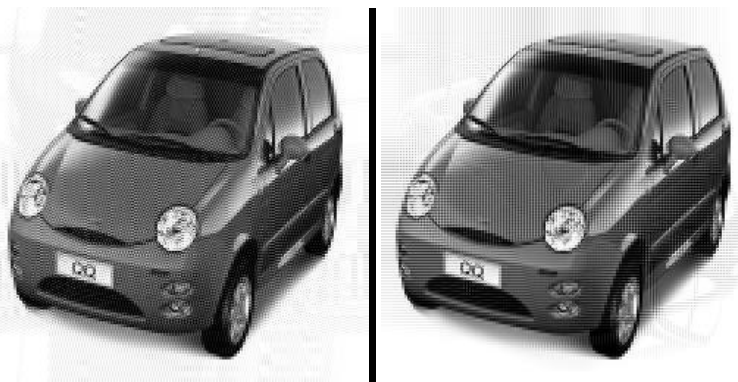

Figure 7c, d-Matted Greyscales (Haar, KWT)
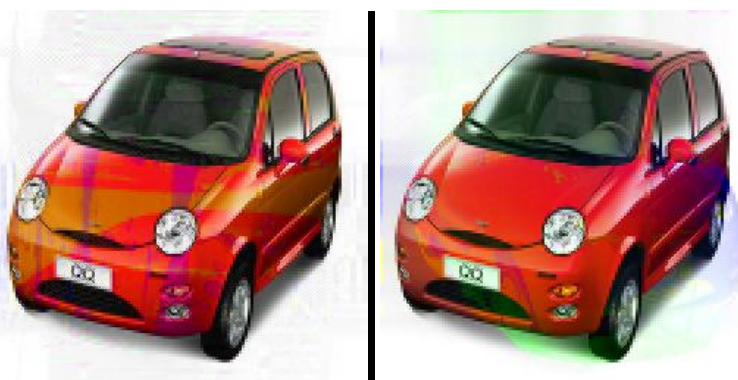

Figure 7e, f-Reconstructed Colour (Haar, KWT)

Figure 7 shows the image of a red car. Once again this is a relatively simple image with all details concentrated solely in the foreground. This image is more complex than that of the ball shown in figure 6. For this image, the matted greyscales look acceptable for both transforms, however a subjective evaluation of the quality of the reconstructed colour images shows the image created using the KWT to be of superior quality. The discolourations in the reconstructed colour image obtained using the Haar transform are very prominent.
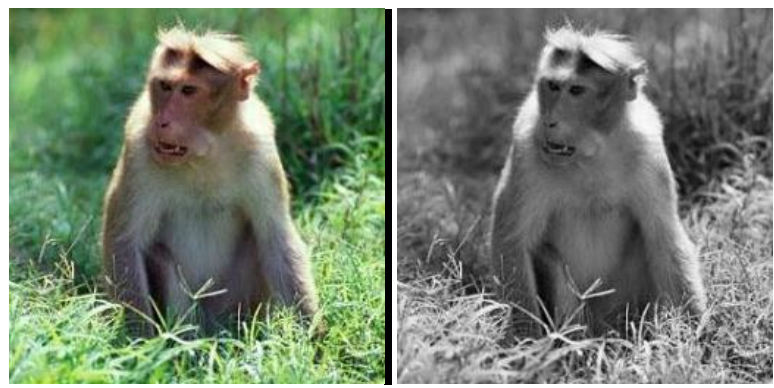

Figure 8a, b - Original Colour, Original Greyscale

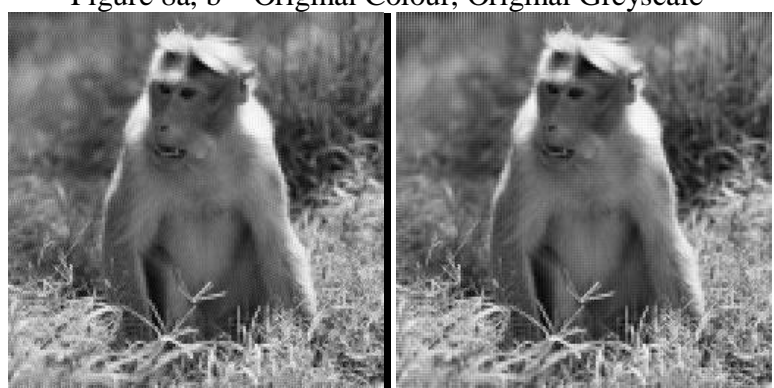

Figure 8c, d-Matted Greyscales (Haar, KWT)

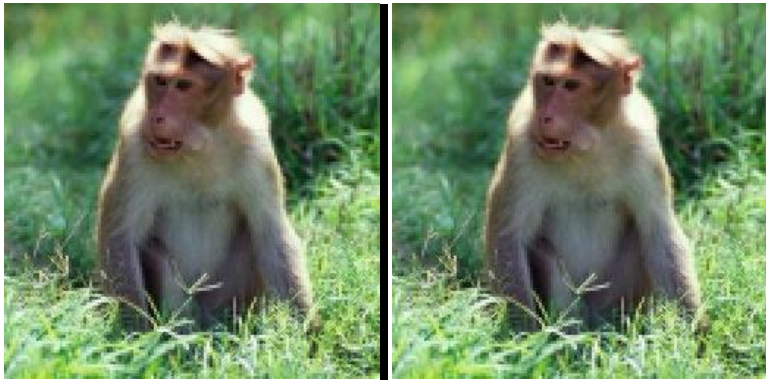

Figure 8e, f - Reconstructed Colour (Haar, KWT)

Figure 8 shows a more complex image than the ones seen till now. This image has both foreground and background detail. The results obtained, using either transform, for this image are similar. Both performed exceedingly well and neither resulted in any extreme discolourations in the reconstructed colour images. The matted greyscale images were also highly acceptable with minimal distortions.

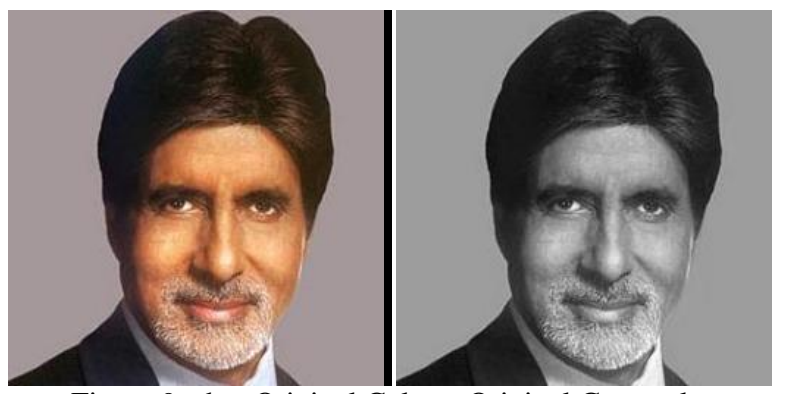

Figure 9a, b - Original Colour, Original Greyscale

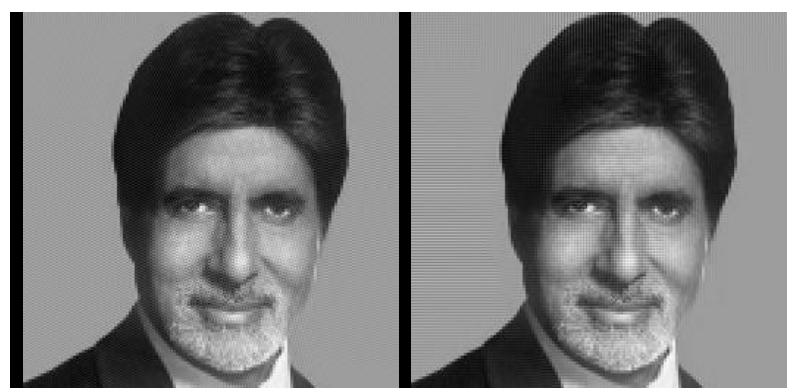

Figure 9c, d-Matted Greyscales (Haar, KWT)

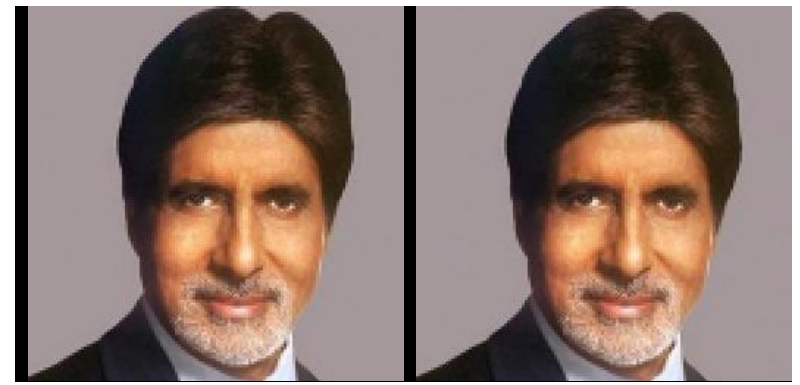

Figure 9e, f - Reconstructed Colour (Haar, KWT)

Finally, figure 9 shows a face. It should be noted that both matted greyscale images have a black strip of pixels on the left which is absent in the original greyscale image, and that this strip of pixels persist even in the reconstructed colour images. Both reconstructed colour images are of similar quality and are acceptable.

The MSE for both the matted greyscale images and the reconstructed colour images using both transforms for the images shown in figures 6 to 9 are given in table 3 and table 4 respectively. Figures 10 and 11 illustrate the same results graphically. 


\begin{tabular}{|c|c|c|}
\hline & MSE (Haar) & MSE (KWT) \\
\hline Ball & 368.8388 & 359.4922 \\
\hline Car & 764.5332 & 741.3582 \\
\hline Monkey & 274.749 & 274.4384 \\
\hline Face & 228.1034 & 228.375 \\
\hline \multicolumn{3}{|c}{ Table 3 - Greyscale MSE }
\end{tabular}

\begin{tabular}{|c|c|c|}
\hline & MSE (Haar) & MSE (KWT) \\
\hline Ball & 173.84852 & 196.052 \\
\hline Car & 497.4836 & 561.1308 \\
\hline Monkey & 158.2638 & 158.675 \\
\hline Face & 45.67854 & 45.83958 \\
\hline \multicolumn{3}{|c}{ Table 4 - Colour MSE }
\end{tabular}

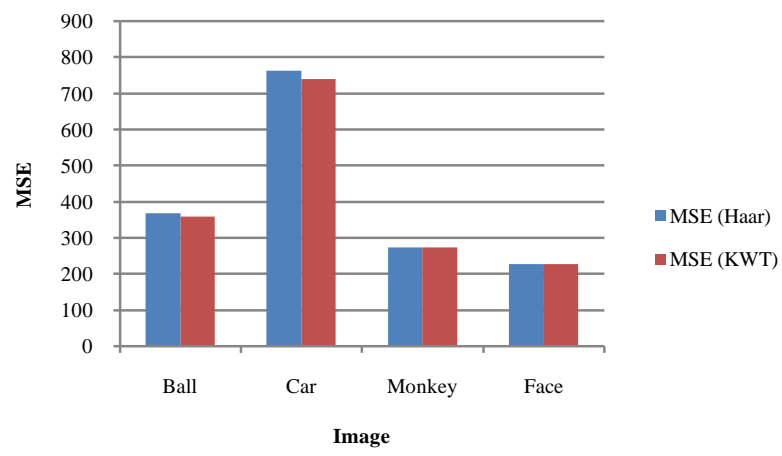

Figure 10 - Graphical Representation of Greyscale MSE

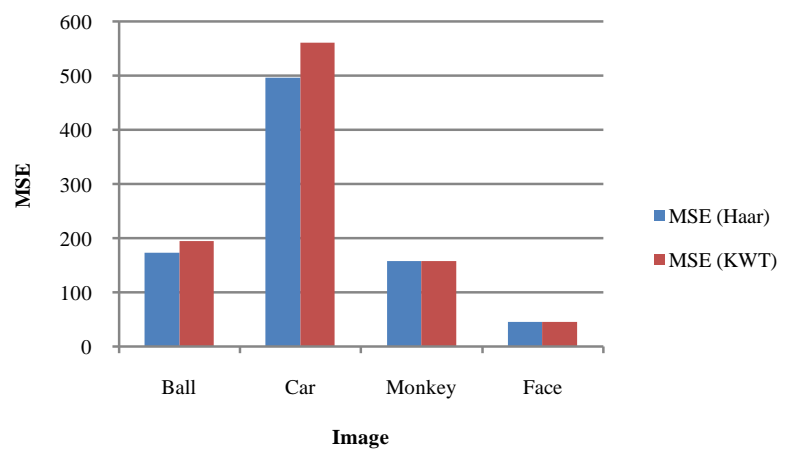

Figure 11 - Graphical Representation of Colour MSE

\section{CONCLUSION}

In conclusion, it can be said that the performance of both the Haar wavelet transform and the KWT using this technique to embed colour information inside a greyscale image is similar when compared objectively using the MSE. However, when the images are subjectively compared, the discolourations caused when the Haar transform is used are more prominent and distracting than when the KWT is used. Thus, the KWT is a good choice for the wavelet transform to be used when implementing this technique, especially with its added advantage of working on all image sizes and not just those images whose sizes are a power of two.

\section{REFERENCES}

[1] H. B. Kekre, Sudeep D. Thepade, Adib Parkar, "Storage of Colour Information in a Greyscale Image using Haar Wavelets and Various Colour Spaces", International Journal of Computer Applications (IJCA) September 2010.
[2] Ricardo L. de Queiroz, Karen M. Braun, "Color to Gray and Back: Color Embedding into Textured Gray Images", 1464 IEEE TRANSACTIONS ON IMAGE PROCESSING, VOL. 15, NO. 6, JUNE 2006.

[3] Son Lam Fung, A. Bouzerdoum, D. Chai, “A novel skin color model in $\mathrm{YCbCr}$ color space and its application to human face detection", In Proc. of International Conference on Image Processing (ICIP-2002), Vol.1, pp. I289-I292.

[4] Hideki Noda, Michiharu Niimi, "Colorization in $\mathrm{YCbCr}$ color space and its application to JPEG images", Pattern Recognition Society Published by Elsevier B.V., Vol.40, number 12, pp.3714-3720, December, 2007.

[5] H. B. Kekre, Sudeep D. Thepade, Archana Athawale, Adib Parkar, "Revisiting 'Color Traits Transfer to Grayscale Images' using Assorted Color Spaces and Pixel Window Sizes," ACM-International Conference and Workshop on Emerging Trends in Technology (ICWET 2010), Thakur College of Engg. And Tech., Mumbai, 26-27 Feb 2010.

[6] H. B. Kekre, Sudeep D. Thepade, "Image Blending in Vista Creation using Kekre's LUV Color Space", In SPIT-IEEE Colloquium, SPIT Mumbai, INDIA, Feb 4-5,2008.

[7] H. B. Kekre, Sudeep D. Thepade, "Improving 'Color to Gray and Back' using Kekre's LUV Color Space”, IEEE International Advanced Computing Conference 2009 (IACC '09), Thapar University, Patiala, INDIA, 6-7 March 2009.

[8] "Boosting Block Truncation Coding using Kekre's LUV Color Space for Image Retrieval", WASET International Journal of Electrical, Computer and System Engineering (IJECSE), Volume 2, No.3, Summer 2008.

[9] H. B. Kekre, Sudeep D. Thepade, Adib Parkar, "A Comparison of Kekre's Fast Search and Exhaustive Search for various Grid Sizes used for Colouring a Greyscale Image," 2nd International Conference on Signal Acquisition and Processing (ICSAP 2010), IACSIT, Bangalore, pp. 53-57, 910 Feb 2010.

[10] Haar, Alfred, "Zur Theorie der orthogonalen Funktionen systeme". (German), Mathematische Annalen, volume 69, No. 3, 1910, pp. 331-371.

[11] Charles K. Chui, "An Introduction to Wavelets", Academic Press, 1992, San Diego, ISBN 0585470901.

[12] E. Reinhard, M. Ashikhmin, B. Gooch, and P. Shirley, "Color transfer between images," IEEE Computer graphics and applications, vol. 21 , no. 5, pp. 34-41, September/October 2001.

[13] D. L. Ruderman, T. W. Cronin, and C. C. Chiao, "Statistics of cone responses to natural images: implications for visual coding," J. Optical Soc. Of America, vol. 15, no. 8, pp. 20362045, 1998

\section{AUTHOR BIOGRAPHIES}

Dr. H. B. Kekre has received B.E. (Hons.) in Telecomm. Engg. from Jabalpur University in 1958, M.Tech (Industrial Electronics) from IIT Bombay in 1960, M.S.Engg. (Electrical Engg.) from University of Ottawa in 1965 and Ph.D. (System Identification) from IIT Bombay in 1970. He has worked Over 35 years as Faculty of Electrical Engineering and then HOD Computer Science and Engg. at IIT Bombay. For last 13 years worked as a Professor in Department of Computer Engg. at Thadomal Shahani Engineering College, Mumbai. He is currently Senior Professor working with Mukesh Patel School of Technology Management and Engineering, SVKM's NMIMS University, Vile Parle(w), Mumbai, INDIA. He ha guided 17 Ph.D.s, 150 M.E./M.Tech Projects and several B.E./B.Tech Projects. 
His areas of interest are Digital Signal processing and Image Processing. He has more than 300 papers in National / International Conferences / Journals to his credit. Recently six students working under his guidance have received best paper awards. Currently he is guiding seven Ph.D. students.

Sudeep D. Thepade has Received B.E.(Computer) degree from North Maharashtra University with Distinction in 2003. M.E. in Computer Engineering from University of Mumbai in 2008 with Distinction, currently Perusing Ph.D. from SVKM's NMIMS University, Mumbai. He has about 08 years of experience in teaching and industry. He was Lecturer in Dept. of Information Technology at Thadomala Shahani Engineering College, Bandra(w), Mumbai for nearly 04 years. Currently working as Assistant Professor in Computer Engineering at Mukesh Patel School of Technology Management and Engineering, SVKM's NMIMS University, Vile Parle(w), Mumbai, INDIA.
$\mathrm{He}$ is member of International Association of Engineers (IAENG) and International Association of Computer Science and Information Technology (IACSIT), Singapore. His areas of interest are Image Processing and Computer Networks. He more than 90 papers in National/International Conferences/Journals to his credit with a Best Paper Award at International Conference SSPCCIN2008 and Second Best Paper Award at ThinkQuest-2009 National Level paper presentation competition for faculty.

Adib Parkar is currently pursuing a Bachelors (B.E.) degree in Computer Science from Thadomal Shahani Engineering College in Mumbai, India. He has been an active IEEE Student Member for 3 years and is also a member of the Computer Society of India. His areas of interest lie in the fields of Image Processing and Artificial Intelligence. 